\title{
Stochastic Oscillators with Quadratic Nonlinearity Using WHEP and HPM Methods
}

\author{
Amnah S. Al-Johani, ${ }^{1,2}$ \\ ${ }^{1}$ Department of Applied Mathematics, College of Science, Northern Borders University, Arar, Saudi Arabia \\ ${ }^{2}$ College of Home Economics, Northern Borders University, Arar, Saudi Arabia \\ Email: xxwhitelinnetxx@hotmail.com
}

Received May 2, 2013; revised June 8, 2013; accepted July 2, 2013

Copyright (C 2013 Amnah S. Al-Johani. This is an open access article distributed under the Creative Commons Attribution License, which permits unrestricted use, distribution, and reproduction in any medium, provided the original work is properly cited.

\begin{abstract}
In this paper, quadratic nonlinear oscillators under stochastic excitation are considered. The Wiener-Hermite expansion with perturbation (WHEP) method and the homotopy perturbation method (HPM) are used and compared. Different approximation orders are considered and statistical moments are computed in the two methods. The two methods show efficiency in estimating the stochastic response of the nonlinear differential equations.
\end{abstract}

Keywords: Nonlinear Stochastic Differential Equations; Wiener-Hermite Expansion; WHEP Technique; Homotopy Perturbation Method

\section{Introduction}

Quadrate oscillation arises through many applied models in applied sciences and engineering when studying oscillatory systems [1]. These systems can be exposed to a lot of uncertainties through the external forces, the damping coefficient, the frequency and/or the initial or boundary conditions. These input uncertainties cause the output solution process to be also uncertain. For most of the cases, getting the probability density function (p.d.f.) of the solution process may be impossible. So, developing approximate techniques through which approximate statistical moments can be obtained, is an important and necessary work.

Since Meecham and his co-workers [2] developed a theory of turbulence involving a truncated Wiener-Hermite expansion (WHE) of the velocity field, many authors studied problems concerning turbulence [3-8]. A lot of general applications in fluid mechanics were also studied in [9-11]. Scattering problems attracted the WHE applications through many authors [12-16]. The nonlinear oscillators were considered as an opened area for the applications of WHE as can be found in [17-23]. There are a lot of applications in boundary value problems [24, 25] and generally in different mathematical studies [2629]. The WHE properties and description of its usage are given in [30].

In HPM technique [31-34], the response of nonlinear differential equations can be obtained analytically as a series solution. The basic idea of the homotopy method is to deform continuously a simple problem (and easy to solve) into the difficult problem under study [35]. The HPM method is a special case of homotopy analysis method (HAM) propounded by Liao in 1992 [36]. The HAM was systematically described in Liao's book in 2003 [37] and was applied by many authors in [38-41]. The HAM method possesses auxiliary parameters and functions which can control the convergence of the obtained series solution.

The stochastic oscillator with cubic nonlinearity (Duffing oscillator) was considered in [17,42]. The nonlinear term is due to the restoring nonlinear force. In some applications, the restoring force is quadratic and it is required to estimate the response in this case. The main goal of this paper is to consider the quadratic nonlinear oscillator under stochastic excitation. The WHEP and HPM methods are used and compared.

This paper is organized as follows. The problem formulation is outlined in Section 2. The WHEP technique is described and applied to the stochastic quadratic oscillator in Section 3. The HPM is outlined in Section 4 and applied also to the quadratic oscillator. A comparison between the two methods is shown in Section 5 .

\section{Problem Formulation}

In this section, the following quadratic nonlinear oscillatory equation is considered: 


$$
\ddot{x}(t ; \omega)+2 w \zeta \dot{x}+w^{2} x+\varepsilon w^{2} x^{2}=F(t ; \omega), t \in[0, T]
$$

under stochastic excitation $F(t ; \omega)$ with deterministic initial conditions

$$
x(0)=x_{0}, \quad \dot{x}(0)=\dot{x}_{0},
$$

where

$w$ : frequency of oscillation,

$\zeta$ : damping coefficient,

$\varepsilon$ : deterministic nonlinearity scale,

$\omega \in(\Omega, \sigma, p)$ : a triple probability space with $\Omega$ as the sample space, $\sigma$ is a $\sigma$-algebra on events in $\Omega$ and $P$ is a probability measure.

\section{WHEP Technique}

The application of the WHE aims at finding a truncated series solution to the solution process of differential equations. The truncated series composes of two major parts; the first is the Gaussian part which consists of the first two terms, while the rest of the series constitute the non-
Gaussian part. In nonlinear cases, there exists always difficulties of solving the resultant set of deterministic integro-differential equations got from the applications of a set of comprehensive averages on the stochastic integrodifferential equation obtained after the direct application of WHE. Many authors introduced different methods to face these obstacles. Among them, the WHEP technique was introduced in [22] using the perturbation technique to solve perturbed nonlinear problems.

The WHE method utilizes the Wiener-Hermite polynomials which are the elements of a complete set of statistically orthogonal random functions [30]. The WienerHermite polynomial $H^{(i)}\left(t_{1}, t_{2}, \cdots, t_{i}\right)$ satisfies the following recurrence relation:

$$
\begin{aligned}
& H^{(i)}\left(t_{1}, t_{2}, \cdots, t_{i}\right)=H^{(i-1)}\left(t_{1}, t_{2}, \cdots, t_{i-1}\right) \cdot H^{(1)}\left(t_{i}\right) \\
& -\sum_{m=1}^{i-1} H^{(i-2)}\left(t_{i_{1}}, t_{i_{2}}, \cdots, t_{i_{i-2}}\right) \cdot \delta\left(t_{i-m}-t_{i}\right), i \geq 2
\end{aligned}
$$

where

$$
\begin{aligned}
& H^{(0)}=1, \quad H^{(1)}(t)=n(t), \quad H^{(2)}\left(t_{1}, t_{2}\right)=H^{(1)}\left(t_{1}\right) \cdot H^{(1)}\left(t_{2}\right)-\delta\left(t_{1}-t_{2}\right), \\
& H^{(3)}\left(t_{1}, t_{2}, t_{3}\right)=H^{(2)}\left(t_{1}, t_{2}\right) \cdot H^{(1)}\left(t_{3}\right)-H^{(1)}\left(t_{1}\right) \cdot \delta\left(t_{2}-t_{3}\right)-H^{(1)}\left(t_{2}\right) \cdot \delta\left(t_{1}-t_{3}\right), \\
& H^{(4)}\left(t_{1}, t_{2}, t_{3}, t_{4}\right)=H^{(3)}\left(t_{1}, t_{2}, t_{3}\right) \cdot H^{(1)}\left(t_{4}\right)-H^{(2)}\left(t_{1}, t_{2}\right) \cdot \delta\left(t_{3}-t_{4}\right)-H^{(2)}\left(t_{1}, t_{3}\right) \cdot \delta\left(t_{2}-t_{4}\right)-H^{(2)}\left(t_{2}, t_{3}\right) \cdot \delta\left(t_{1}-t_{4}\right),
\end{aligned}
$$

in which $n(t)$ is the white noise with the following statistical properties

$$
E n(t)=0, \quad E n\left(t_{1}\right) \cdot n\left(t_{2}\right)=\delta\left(t_{1}-t_{2}\right),
$$

where $\delta($.$) is the Dirac delta function and E$ denotes the ensemble average operator. i.e.

The Wiener-Hermite set is a statistically orthogonal set,

$$
\begin{aligned}
G(t ; \omega)= & G^{(0)}(t)+\int_{-\infty}^{\infty} G^{(1)}\left(t ; t_{1}\right) H^{(1)}\left(t_{1}\right) \mathrm{d} t_{1}+\int_{-\infty}^{\infty} \int_{-\infty}^{\infty} G^{(2)}\left(t ; t_{1}, t_{2}\right) H^{(2)}\left(t_{1}, t_{2}\right) \mathrm{d} t_{1} \mathrm{~d} t_{2} \\
& +\int_{-\infty}^{\infty} \int_{-\infty}^{\infty} \int_{-\infty}^{\infty} G^{(3)}\left(t ; t_{1}, t_{2}, t_{3}\right) H^{(3)}\left(t_{1}, t_{2}, t_{3}\right) \mathrm{d} t_{1} \mathrm{~d} t_{2} \mathrm{~d} t_{3}+\cdots
\end{aligned}
$$

where the first two terms are the Gaussian part of $G(t ; \omega)$. The rest of the terms in the expansion represent the non-Gaussian part of $G(t ; \omega)$. The average of $G(t ; \omega)$ is

$$
\mu_{G}=E G(t ; \omega)=G^{(0)}(t)
$$

The covariance of $G(t ; \omega)$ is

$$
\begin{aligned}
& \operatorname{Cov}(G(t ; \omega), G(\tau ; \omega))=E\left(G(t ; \omega)-\mu_{G}(t)\right)\left(G(\tau ; \omega)-\mu_{G}(\tau)\right) \\
& =\int_{-\infty}^{\infty} G^{(1)}\left(t ; t_{1}\right) G^{(1)}\left(\tau, t_{1}\right) \mathrm{d} t_{1}+2 \int_{-\infty}^{\infty} \int_{-\infty}^{\infty} G^{(2)}\left(t ; t_{1}, t_{2}\right) G^{(2)}\left(\tau, t_{1}, t_{2}\right) \mathrm{d} t_{1} \mathrm{~d} t_{2} \\
& +2 \int_{-\infty}^{\infty} \int_{-\infty}^{\infty} \int_{-\infty}^{\infty} G^{(3)}\left(t ; t_{1}, t_{2}, t_{3}\right)\left[G^{(3)}\left(\tau, t_{1}, t_{2}, t_{3}\right)+G^{(3)}\left(\tau, t_{1}, t_{3}, t_{2}\right)+G^{(3)}\left(\tau, t_{2}, t_{3}, t_{1}\right)\right] \mathrm{d} t_{1} \mathrm{~d} t_{2} \mathrm{~d} t_{3}+\cdots
\end{aligned}
$$


The variance of $G(t ; \omega)$ is

$$
\begin{aligned}
& \operatorname{Var} G(t ; \omega)=E\left(G(t ; \omega)-\mu_{G}(t)\right)^{2}=\int_{-\infty}^{\infty}\left[G^{(1)}\left(t ; t_{1}\right)\right]^{2} \mathrm{~d} t_{1}+2 \int_{-\infty}^{\infty} \int_{-\infty}^{\infty}\left[G^{(2)}\left(t ; t_{1}, t_{2}\right)\right]^{2} \mathrm{~d} t_{1} \mathrm{~d} t_{2} \\
& +2 \int_{-\infty}^{\infty} \int_{-\infty}^{\infty} \int_{-\infty}^{\infty}\left[G^{(3)}\left(t ; t_{1}, t_{2}, t_{3}\right)\right]^{2} \mathrm{~d} t_{1} \mathrm{~d} t_{2} \mathrm{~d} t_{3}+2 \int_{-\infty}^{\infty} \int_{-\infty}^{\infty} \int_{-\infty}^{\infty}\left[G^{(3)}\left(t, t_{1}, t_{2}, t_{3}\right) G^{(3)}\left(t, t_{1}, t_{3}, t_{2}\right)\right] \mathrm{d} t_{1} \mathrm{~d} t_{2} \mathrm{~d} t_{3} \\
& +2 \int_{-\infty}^{\infty} \int_{-\infty}^{\infty} \int_{-\infty}^{\infty}\left[G^{(3)}\left(t, t_{1}, t_{2}, t_{3}\right) G^{(3)}\left(t, t_{2}, t_{3}, t_{1}\right)\right] \mathrm{d} t_{1} \mathrm{~d} t_{2} \mathrm{~d} t_{3}+\cdots
\end{aligned}
$$

The WHE method can be elementary used in solving stochastic differential equations by expanding the solution process as well as the stochastic input processes via the WHE. The resultant equation is more complex than the original one due to being a stochastic integro-differential equation. Taking a set of ensemble averages together with using the statistical properties of the WH polynomials, a set of deterministic integro-differential equations are obtained in the deterministic kernels $G^{(i)}(t ; \omega), i=0,1,2, \cdots$. To obtain an approximate solutions for these deterministic kernels, one can use perturbation theory in the case of having a perturbed system depending on, say, $\varepsilon$. Expanding the kernels as a power series of $\varepsilon$, another set of simpler iterative equations in the kernel series components are obtained. This is the main algorithm of the WHEP technique. The technique was successfully applied to several nonlinear stochastic equations; see [20,22,23,25].

The WHEP technique can be applied on linear or nonlinear perturbed systems described by ordinary or partial differential equations. The solution can be modified in the sense that additional parts of the Wiener-
Hermite expansion can always be taken into considerations and the required order of approximations can always be made. It can be even run through a package if it is coded in some sort of symbolic languages.

\section{Case-Study}

The quadratic nonlinear oscillatory problem, Equation (1) under stochastic excitation $F(t ; \omega)$ with deterministic initial conditions is solved using WHEP technique. The solution process takes the following form:

$$
\begin{aligned}
& x(t ; \omega)=x^{(0)}(t)+\int_{-\infty}^{\infty} x^{(1)}\left(t ; t_{1}\right) H^{(1)}\left(t_{1}\right) \mathrm{d} t_{1} \\
& +\int_{-\infty}^{\infty} \int_{-\infty}^{\infty} x^{(2)}\left(t ; t_{1}, t_{2}\right) H^{(2)}\left(t_{1}, t_{2}\right) \mathrm{d} t_{1} \mathrm{~d} t_{2} \\
& +\int_{-\infty}^{\infty} \int_{-\infty}^{\infty} \int_{-\infty}^{\infty} x^{(3)}\left(t ; t_{1}, t_{2}, t_{3}\right) H^{(3)}\left(t_{1}, t_{2}, t_{3}\right) \mathrm{d} t_{1} \mathrm{~d} t_{2} \mathrm{~d} t_{3}+\cdots
\end{aligned}
$$

Applying the WHEP technique, the following equations in the deterministic kernels are obtained:

$$
\begin{aligned}
& L x^{(0)}(t)+\varepsilon w^{2}\left(x^{(0)}(t)\right)^{2}+\varepsilon w^{2} \int_{-\infty}^{\infty}\left(x^{(1)}\left(t ; t_{1}\right)\right)^{2} \mathrm{~d} t_{1}+2 \varepsilon w^{2} \int_{-\infty}^{\infty} \int_{-\infty}^{\infty}\left(x^{(2)}\left(t ; t_{1}, t_{2}\right)\right)^{2} \mathrm{~d} t_{1} \mathrm{~d} t_{2} \\
& +2 \varepsilon w^{2}\left[\int_{-\infty}^{\infty} \int_{-\infty}^{\infty} \int_{-\infty}^{\infty}\left(x^{(3)}\left(t ; t_{1}, t_{2}, t_{3}\right)\right)^{2} \mathrm{~d} t_{1} \mathrm{~d} t_{2} \mathrm{~d} t_{3}+\int_{-\infty}^{\infty} \int_{-\infty}^{\infty} \int_{-\infty}^{\infty} x^{(3)}\left(t ; t_{1}, t_{2}, t_{3}\right) x^{(3)}\left(t, t_{2}, t_{3}, t_{1}\right) \mathrm{d} t_{1} \mathrm{~d} t_{2} \mathrm{~d} t_{3}\right. \\
& \left.+\int_{-\infty}^{\infty} \int_{-\infty}^{\infty} \int_{-\infty}^{\infty} x^{(3)}\left(t ; t_{1}, t_{2}, t_{3}\right) x^{(3)}\left(t, t_{1}, t_{3}, t_{2}\right) \mathrm{d} t_{1} \mathrm{~d} t_{2} \mathrm{~d} t_{3}\right]=F^{(0)}(t) \\
& L x^{(1)}\left(t, t_{1}\right)+2 \varepsilon w^{2} x^{(0)}(t) x^{(1)}\left(t, t_{1}\right)+4 \varepsilon w^{2} \int_{-\infty}^{\infty} x^{(1)}\left(t ; t_{2}\right) x^{(2)}\left(t ; t_{1}, t_{2}\right) \mathrm{d} t_{2} \\
& +4 \varepsilon w^{2} \int_{-\infty}^{\infty} \int_{-\infty}^{\infty} x^{(1)}\left(t, t_{2}\right) x^{(2)}\left(t ; t_{1}, t_{2}\right) \mathrm{d} t_{2}+8 \varepsilon w^{2}\left[\int_{-\infty}^{\infty} \int_{-\infty}^{\infty} x^{(2)}\left(t ; t_{2}, t_{3}\right) x^{(3)}\left(t ; t_{1}, t_{2}, t_{3}\right) \mathrm{d} t_{2} \mathrm{~d} t_{3}\right. \\
& \left.+4 \varepsilon w^{2} \int_{-\infty}^{\infty} \int_{-\infty}^{\infty} x^{(2)}\left(t ; t_{2}, t_{3}\right) x^{(3)}\left(t ; t_{2}, t_{3}, t_{1}\right) \mathrm{d} t_{2} \mathrm{~d} t_{3}\right]=F^{(1)}\left(t, t_{1}\right)
\end{aligned}
$$




$$
\begin{aligned}
& L x^{(2)}\left(t, t_{1}, t_{2}\right)+\varepsilon w^{2}\left[2 x^{(0)}(t) x^{(2)}\left(t, t_{1}, t_{2}\right)+x^{(1)}\left(t, t_{1}\right) x^{(1)}\left(t, t_{2}\right)+4 \int_{-\infty}^{\infty} x^{(2)}\left(t ; t_{1}, t_{3}\right) x^{(2)}\left(t ; t_{2}, t_{3}\right) \mathrm{d} t_{3}\right. \\
& +2 \int_{-\infty}^{\infty} x^{(1)}\left(t ; t_{3}\right) x^{(3)}\left(t ; t_{1}, t_{3}, t_{2}\right) \mathrm{d} t_{3}+2 \int_{-\infty}^{\infty} x^{(1)}\left(t ; t_{3}\right) x^{(3)}\left(t ; t_{2}, t_{3}, t_{1}\right) \mathrm{d} t_{3}+2 \int_{-\infty}^{\infty} x^{(1)}\left(t ; t_{3}\right) x^{(3)}\left(t ; t_{1}, t_{2}, t_{3}\right) \mathrm{d} t_{3} \\
& +2 \int_{-\infty}^{\infty} \int_{-\infty}^{\infty} x^{(3)}\left(t ; t_{2}, t_{5}, t_{6}\right) x^{(3)}\left(t ; t_{1}, t_{6}, t_{5}\right) \mathrm{d} t_{5} \mathrm{~d} t_{6}+4 \int_{-\infty}^{\infty} \int_{-\infty}^{\infty} x^{(3)}\left(t ; t_{2}, t_{5}, t_{6}\right) x^{(3)}\left(t ; t_{1}, t_{5}, t_{6}\right) \mathrm{d} t_{5} \mathrm{~d} t_{6} \\
& +3 \int_{-\infty}^{\infty} \int_{-\infty}^{\infty} x^{(3)}\left(t ; t_{2}, t_{5}, t_{6}\right) x^{(3)}\left(t ; t_{5}, t_{6}, t_{1}\right) \mathrm{d} t_{5} \mathrm{~d} t_{6}+2 \int_{-\infty}^{\infty} \int_{-\infty}^{\infty} x^{(3)}\left(t ; t_{1}, t_{5}, t_{6}\right) x^{(3)}\left(t ; t_{2}, t_{6}, t_{5}\right) \mathrm{d} t_{5} \mathrm{~d} t_{6} \\
& +3 \int_{-\infty}^{\infty} \int_{-\infty}^{\infty} x^{(3)}\left(t ; t_{1}, t_{5}, t_{6}\right) x^{(3)}\left(t ; t_{5}, t_{6}, t_{2}\right) \mathrm{d} t_{5} \mathrm{~d} t_{6}+\int_{-\infty}^{\infty} \int_{-\infty}^{\infty} x^{(3)}\left(t ; t_{1}, t_{6}, t_{5}\right) x^{(3)}\left(t ; t_{5}, t_{6}, t_{2}\right) \mathrm{d} t_{5} \mathrm{~d} t_{6} \\
& \left.+2 \int_{-\infty}^{\infty} \int_{-\infty}^{\infty} x^{(3)}\left(t ; t_{5}, t_{6}, t_{2}\right) x^{(3)}\left(t ; t_{5}, t_{6}, t_{1}\right) \mathrm{d} t_{5} \mathrm{~d} t_{6}+\int_{-\infty}^{\infty} \int_{-\infty}^{\infty} x^{(3)}\left(t ; t_{2}, t_{6}, t_{5}\right) x^{(3)}\left(t ; t_{5}, t_{6}, t_{1}\right) \mathrm{d} t_{5} \mathrm{~d} t_{6}\right]=F^{(2)}\left(t, t_{1}, t_{2}\right) \\
& L x^{(3)}\left(t, t_{1}, t_{2}, t_{3}\right)+L x^{(3)}\left(t, t_{1}, t_{3}, t_{2}\right)+L x^{(3)}\left(t, t_{2}, t_{3}, t_{1}\right)+\varepsilon w^{2}\left[2 x^{(0)}(t)\left[x^{(3)}\left(t, t_{1}, t_{2}, t_{3}\right)+x^{(3)}\left(t, t_{1}, t_{3}, t_{2}\right)+\right] x^{(3)}\left(t, t_{2}, t_{3}, t_{1}\right)\right. \\
& +4 \int_{-\infty}^{\infty} x^{(2)}\left(t ; t_{4}, t_{1}\right) x^{(3)}\left(t ; t_{4}, t_{3}, t_{2}\right) \mathrm{d} t_{4}+4 \int_{-\infty}^{\infty} x^{(2)}\left(t ; t_{4}, t_{1}\right) x^{(3)}\left(t ; t_{4}, t_{2}, t_{3}\right) \mathrm{d} t_{4}+4 \int_{-\infty}^{\infty} x^{(2)}\left(t ; t_{4}, t_{2}\right) x^{(3)}\left(t ; t_{4}, t_{3}, t_{1}\right) \mathrm{d} t_{4} \\
& +4 \int_{-\infty}^{\infty} x^{(2)}\left(t ; t_{4}, t_{2}\right) x^{(3)}\left(t ; t_{4}, t_{1}, t_{3}\right) \mathrm{d} t_{4}+4 \int_{-\infty}^{\infty} x^{(2)}\left(t ; t_{4}, t_{3}\right) x^{(3)}\left(t ; t_{4}, t_{1}, t_{2}\right) \mathrm{d} t_{4}+4 \int_{-\infty}^{\infty} x^{(2)}\left(t ; t_{4}, t_{3}\right) x^{(3)}\left(t ; t_{4}, t_{2}, t_{1}\right) \mathrm{d} t_{4} \\
& \left.+4 \int_{-\infty}^{\infty} x^{(2)}\left(t ; t_{4}, t_{1}\right) x^{(3)}\left(t ; t_{3}, t_{2}, t_{4}\right) \mathrm{d} t_{4}+4 \int_{-\infty}^{\infty} x^{(2)}\left(t ; t_{4}, t_{2}\right) x^{(3)}\left(t ; t_{3}, t_{1}, t_{4}\right) \mathrm{d} t_{4}+4 \int_{-\infty}^{\infty} x^{(2)}\left(t ; t_{4}, t_{3}\right) x^{(3)}\left(t ; t_{1}, t_{2}, t_{4}\right) \mathrm{d} t_{4}\right] \\
& =G^{(3)}\left(t, t_{1}, t_{2}, t_{3}\right)+G^{(3)}\left(t, t_{1}, t_{3}, t_{2}\right)+G^{(3)}\left(t, t_{2}, t_{3}, t_{1}\right)
\end{aligned}
$$

Let us take the simple case of evaluating the only Gaussian part (first order approximation) of the solution process of the previous case study, mainly

$$
x(t ; \omega)=x^{(0)}(t)+\int_{-\infty}^{\infty} x^{(1)}\left(t ; t_{1}\right) H^{(1)}\left(t_{1}\right) \mathrm{d} t_{1} .
$$

In this case, the governing equations are

$$
\begin{gathered}
L x^{(0)}(t)+\varepsilon w^{2}\left[\left[x^{(0)}(t)\right]^{2}+\int_{-\infty}^{\infty}\left[x^{(1)}\left(t ; t_{1}\right)\right]^{2} \mathrm{~d} t_{1}\right]=G^{(0)}(t) \\
L x^{(1)}\left(t, t_{1}\right)+\varepsilon w^{2} 2 x^{(0)}(t) x^{(1)}\left(t, t_{1}\right)=G^{(1)}\left(t, t_{1}\right)
\end{gathered}
$$

The ensemble average is

$$
\mu_{x}(t)=x^{(0)}(t)
$$

and the variance is

$$
\sigma_{x}^{2}(t)=\int_{-\infty}^{\infty}\left[x^{(1)}\left(t ; t_{1}\right)\right]^{2} \mathrm{~d} t_{1}
$$

It has to be noticed that all the previous equations are deterministic linear ones in the general form $\ddot{x}+2 w \xi \dot{x}+w^{2} x=F(t)$ with deterministic initial conditions $x(0)=x_{0}, \dot{x}(0)=\dot{x}_{0}$. It has the general solution

$$
x(t)=x_{0} \phi_{1}(t)+\dot{x}_{0} \phi_{2}+\int_{0}^{t} h(t-s) F(s) \mathrm{d} s
$$

In which we have

$$
\begin{aligned}
& h(t)=\frac{1}{w \sqrt{1-\xi^{2}}} \mathrm{e}^{-w \xi t} \sin w \sqrt{1-\xi^{2}} t, \\
& \phi_{1}(t)=\frac{\xi+\sqrt{\xi^{2}-1}}{2 \sqrt{\xi^{2}-1}} \mathrm{e}^{m t}+\frac{-\xi+\sqrt{\xi^{2}-1}}{2 \sqrt{\xi^{2}-1}} \mathrm{e}^{q t}, \\
& \phi_{2}(t)=\frac{1}{2 w \sqrt{\xi^{2}-1}}\left[\mathrm{e}^{m t}-\mathrm{e}^{q t}\right],
\end{aligned}
$$

where

$$
m=-w \xi+w \sqrt{\xi^{2}-1}, \quad q=-w \xi-w \sqrt{\xi^{2}-1} .
$$

When adding the first term in the non-Gaussian part (the second approximation) of the solution process of the previous case study, mainly

$$
\begin{aligned}
x(t ; \omega)= & x^{(0)}(t)+\int_{-\infty}^{\infty} x^{(1)}\left(t ; t_{1}\right) H^{(1)}\left(t_{1}\right) \mathrm{d} t_{1} \\
& +\int_{-\infty}^{\infty} \int_{-\infty}^{\infty} x^{(2)}\left(t ; t_{1}, t_{2}\right) H^{(2)}\left(t_{1}, t_{2}\right) \mathrm{d} t_{1} \mathrm{~d} t_{2}
\end{aligned}
$$


the governing equations become

$$
\begin{aligned}
& L^{(0)}(t)+\varepsilon w^{2}\left[\left[x^{(0)}(t)\right]^{2}+\int_{-\infty}^{\infty}\left[x^{(1)}\left(t ; t_{1}\right)\right]^{2} \mathrm{~d} t_{1}\right] \\
& +2 \varepsilon w^{2} \int_{-\infty}^{\infty} \int_{-\infty}^{\infty}\left[x^{(2)}\left(t ; t_{1}, t_{2}\right)\right]^{2} \mathrm{~d} t_{1} \mathrm{~d} t_{2}=G^{(0)}(t) \\
& \\
& \quad x^{(1)}\left(t, t_{1}\right)+2 \varepsilon w^{2} x^{(0)}(t) x^{(1)}\left(t, t_{1}\right) \\
& +4 \varepsilon w^{2} \int_{-\infty}^{\infty} x^{(1)}\left(t ; t_{2}\right) x^{(2)}\left(t ; t_{1}, t_{2}\right) \mathrm{d} t_{2}=G^{(1)}\left(t, t_{1}\right) \\
& L x^{(2)}\left(t, t_{1}, t_{2}\right) \\
& +\varepsilon w^{2}\left[2 x^{(0)}(t) x^{(2)}\left(t, t_{1}, t_{2}\right)+x^{(1)}\left(t, t_{1}\right) x^{(1)}\left(t, t_{2}\right)\right. \\
& \left.+4 \int_{-\infty}^{\infty} x^{(2)}\left(t ; t_{1}, t_{3}\right) x^{(2)}\left(t ; t_{2}, t_{3}\right) \mathrm{d} t_{3}\right]=G^{(2)}\left(t, t_{1}, t_{2}\right)
\end{aligned}
$$

The ensemble average is still got by Equation (19) while the variance is got as

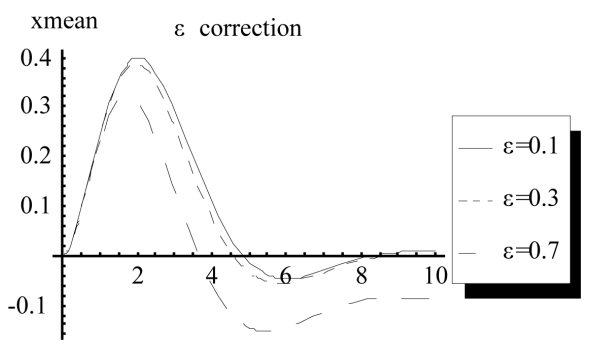

(a)

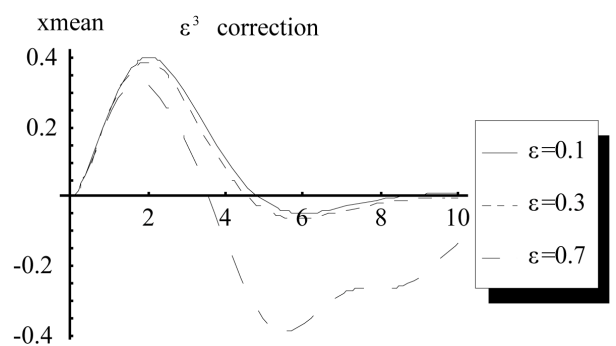

(c)

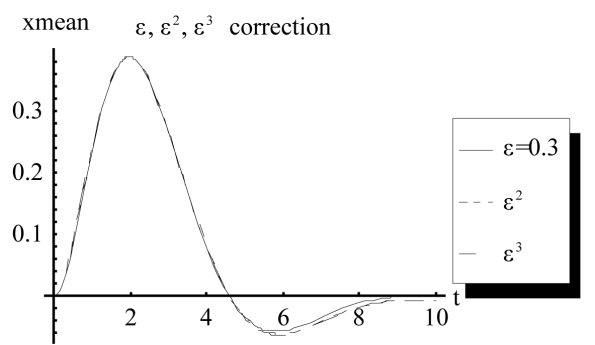

(e)

$$
\sigma_{x}^{2}(t)=\int_{-\infty}^{\infty}\left[x^{(1)}\left(t ; t_{1}\right)\right]^{2} \mathrm{~d} t_{1}+2 \int_{-\infty}^{\infty} \int_{-\infty}^{\infty}\left[x^{(2)}\left(t ; t_{1}, t_{2}\right)\right]^{2} \mathrm{~d} t_{1} \mathrm{~d} t_{2}
$$

The WHEP technique uses the following expansion for its deterministic kernels as corrections made under each approximation order.

$$
x^{(i)}(t)=x_{0}^{(i)}+\varepsilon x_{1}^{(i)}+\varepsilon^{2} x_{2}^{(i)}+\varepsilon^{3} x_{3}^{(i)}+\cdots, i=0,1,2,3, .
$$

\section{Example:}

Let us take $F(t ; q)=\mathrm{e}^{-t}+\varepsilon \delta(t ; \omega), \quad \varepsilon=0.3$

in the previous case-study and then solving using the WHEP technique. The following results are obtained, see Figures 1-3.

\section{The Homotopy Perturbation Method (HPM)}

In this technique, a parameter $p \in[0,1]$ is embedded in a homotopy function $v(r, p): \phi \times[0,1] \rightarrow \Re$ which satisfies

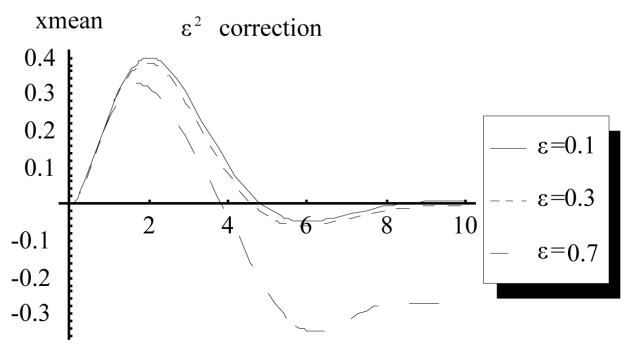

(b)

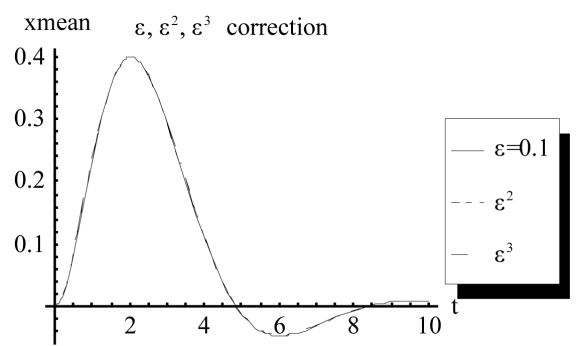

(d)

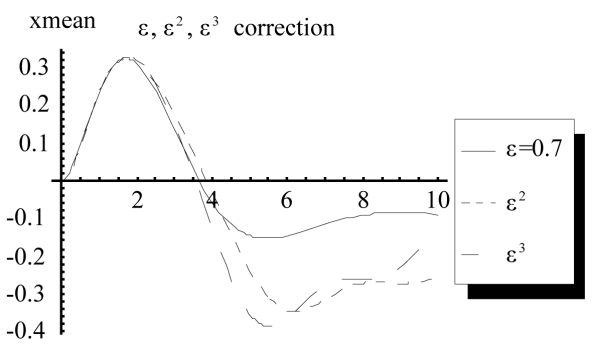

(f)

Figure 1. (a) The first order approximation of the mean at $\varepsilon$ correction for different correction levels; (b) The first order approximation of the mean at $\varepsilon^{2}$ correction for different correction levels; (c) The first order approximation of the mean at $\varepsilon^{3}$ correction; (d) The first order approximation of the mean at $\varepsilon, \varepsilon^{2}, \varepsilon^{3}$ correction; (e) The first order approximation of the mean at $\varepsilon, \varepsilon^{2}, \varepsilon^{3}$ correction; (f) The first order approximation of the mean at $\varepsilon, \varepsilon^{2}, \varepsilon^{3}$ correction. 


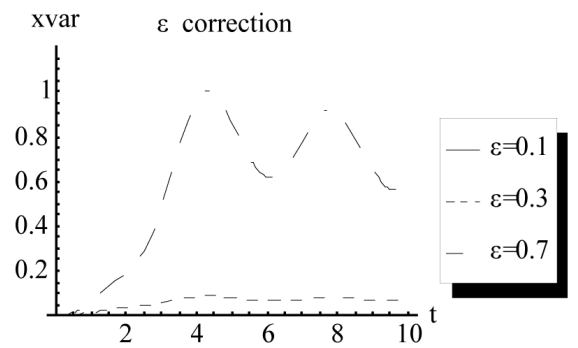

(a)

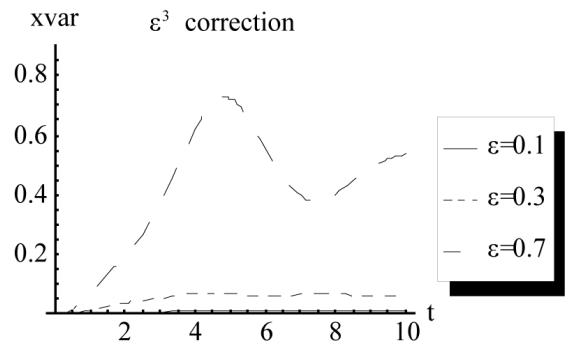

(c)

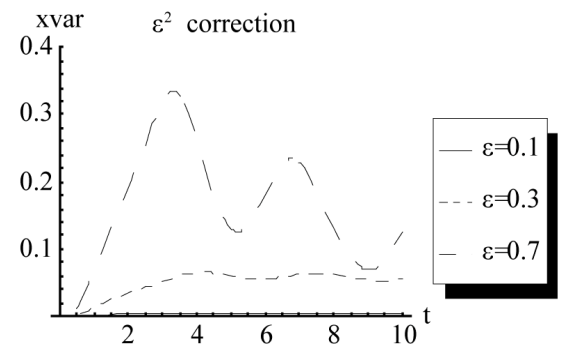

(b)

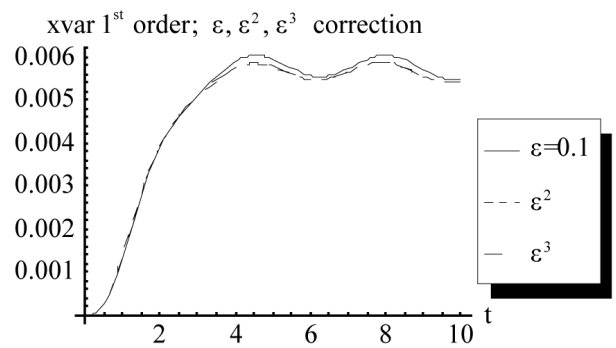

(d)

Figure 2. (a) The first order approximation of the variance at $\varepsilon$ correction for different correction levels; (b) The first order approximation of the variance at $\varepsilon^{2}$ Correction for different correction levels; (c) The first order approximation of the variance at $\varepsilon^{3}$ correction for different correction levels; (d) The first order approximation of the variance at. $\varepsilon, \varepsilon^{2}, \varepsilon^{3}$ correction.

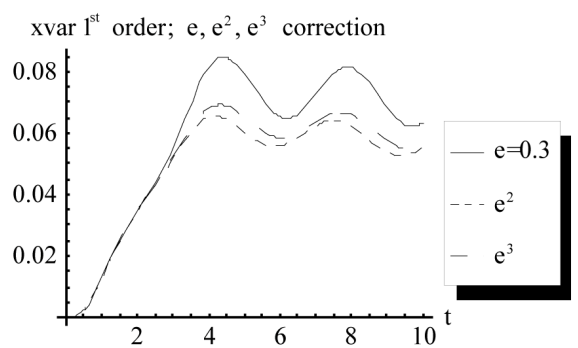

(a)

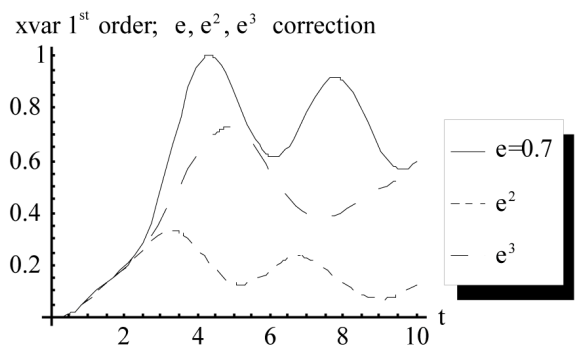

(b)

Figure 3. (a) The first order approximation of the variance at $\varepsilon, \varepsilon^{2}, \varepsilon^{3}$ correction; (b) The first order approximation of the variance at $\varepsilon, \varepsilon^{2}, \varepsilon^{3}$ correction.

$$
H(v, p)=(1-p)\left[L(v)-L\left(u_{0}\right)\right]+p[A(v)-f(r)]=0
$$

where $u_{0}$ is an initial approximation to the solution of the equation

$$
A(u)-f(r)=0, r \in \phi
$$

with boundary conditions

$$
B\left(u, \frac{\partial u}{\partial n}\right)=0, r \in \Gamma
$$

in which $A$ is a nonlinear differential operator which can be decompose into a linear operator $L$ and a nonlinear operator $N, B$ is a boundary operator, $f(r)$ is a known analytic function and $\Gamma$ is the boundary of $\phi$. The homotopy introduces a continuously deformed solution for the case of $p=0, L(v)-L\left(u_{0}\right)=0$, to the case of $p$ $=1, A(v)-f(r)=0$, which is the original Equation (30). This is the basic idea of the homotopy method which is to deform continuously a simple problem (and easy to solve) into the difficult problem under study [35].

The basic assumption of the HPM method is that the solution of the original Equation (29) can be expanded as a power series in $p$ as:

$$
v=v_{0}+p v_{1}+p^{2} v_{2}+p^{3} v_{3}+\cdots
$$

Now, setting $p=1$, the approximate solution of Equation (23) is obtained as:

$$
u=\lim _{p \rightarrow 1} v=v_{0}+v_{1}+v_{2}+v_{3}+\cdots
$$

The rate of convergence of the method depends greatly on the initial approximation $u_{0}$.

The idea of the imbedded parameter can be utilized to solve nonlinear problems by imbedding this parameter to the problem and then forcing it to be unity in the obtained approximate solution if converge can be assured. A simple technique enables the extension of the applicability of the perturbation methods from small valued ap- 
plications to general ones.

\section{Example}

Considering the same previous example of Sub-Section 3.1.1, one can get the following results w.r.t. homotopy perturbation:

$$
\begin{gathered}
A(x)=L(x)+\varepsilon w^{2} x^{2}, \\
L(x)=\ddot{x}+2 w \varsigma \dot{x}+w^{2} x, \\
N(x)=\varepsilon x^{2}, \\
f(r)=F(t ; \omega) .
\end{gathered}
$$

The homotopy function takes the following form:

$$
H(v, p)=(1-p)\left[L(v)-L\left(u_{0}\right)\right]+p[A(v)-f(r)]=0
$$

or equivalently,

$$
L(v)-L\left(u_{0}\right)+p\left[L\left(u_{0}\right)+\varepsilon w^{2} v^{2}-F(t ; \omega)\right]=0 .
$$

Letting $v=v_{0}+p v_{1}+p^{2} v_{2}+p^{3} v_{3}+\cdots$, substituting in Equation (34) and equating the equal powers of $p$ in both sides of the equation, one can get the following results:

1) $L\left(v_{0}\right)=L\left(y_{0}\right)$, in which one may consider the following simple solution:

$$
v_{0}=y_{0}, \quad y_{0}(0)=x_{0}, \dot{y}_{0}(0)=\dot{x}_{0} .
$$

2) $L\left(v_{1}\right)=F(t ; \omega)-L\left(v_{0}\right)-\varepsilon w^{2} v_{0}^{2}, v_{1}(0)=0, \dot{v}_{1}(0)=0$.

3) $L\left(v_{2}\right)=-2 \varepsilon w^{2} v_{0} v_{1}, v_{2}(0)=0, \dot{v}_{2}(0)=0$.

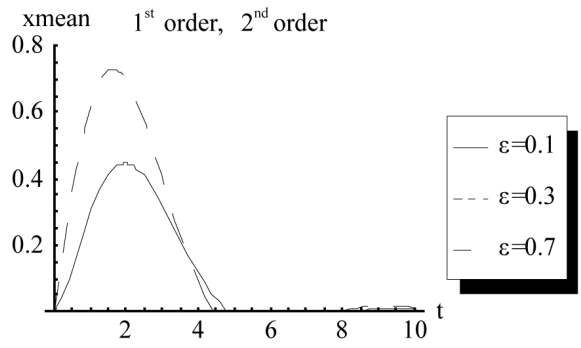

(a)
4) $L\left(v_{3}\right)=-\varepsilon w^{2}\left(v_{1}^{2}+2 v_{0} v_{2}\right), v_{3}(0)=0, \dot{v}_{3}(0)=0$.

5) $L\left(v_{4}\right)=-2 \varepsilon_{1}\left(v_{0} v_{3}+v_{1} v_{2}\right), v_{4}(0)=0, \dot{v}_{4}(0)=0$.

The approximate solution is

$$
x(t ; \omega)=\lim _{p \rightarrow 1} v=v_{0}+v_{1}+v_{2}+v_{3}+\cdots
$$

which can be considered to any approximation order. One can notice that the algorithm of the solution is straight forward and that a lot of flexibilities can be made. For example, we have many choices in guessing the initial approximation together with its initial conditions. For zero initial conditions, we can choose $v_{0}=0$ which leads to:

$$
\begin{aligned}
x(t ; \omega) \cong & x_{5}=v_{0}+v_{1}+v_{2}+v_{3}+v_{4}+v_{5} \\
= & \int_{0}^{t} h(t-s) F(s ; \omega) \mathrm{d} s-\varepsilon w^{2} \int_{0}^{t} h(t-s) v_{1}^{2}(s ; \omega) \mathrm{d} s \\
& -2 \varepsilon w^{2} \int_{0}^{t} h(t-s) v_{1}(s ; \omega) v_{3}(s ; \omega) \mathrm{d} s
\end{aligned}
$$

Figures 4-7 are obtained for $\varsigma=0.5$ : [42].

\section{Comparisons between WHEP and HPM Methods}

Figure [8] shows comparisons between the WHEP and HPM methods for different values of the nonlinearity strength, $\varepsilon$. As the nonlinearity strength increases, the deviation between the two methods is also increasing.

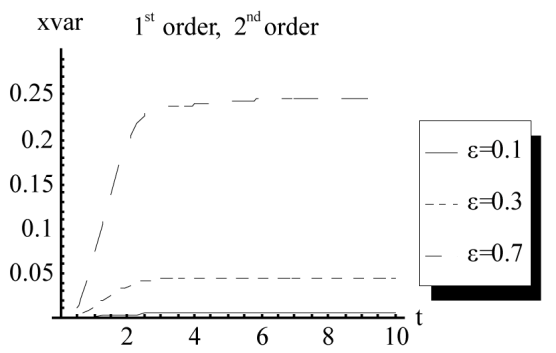

(b)

Figure 4. (a) The first and second order approximation of the mean for different correction levels; (b) The first and second order approximation of the variance at for different correction levels.

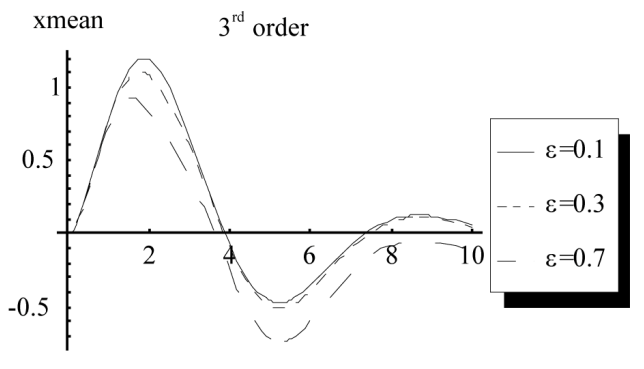

(a)

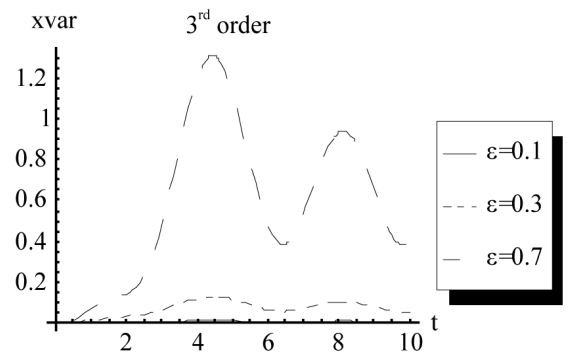

(b)

Figure 5. (a) The third order approximation of the mean for different correction levels; (b) The third order approximation of the variance for different correction levels. 


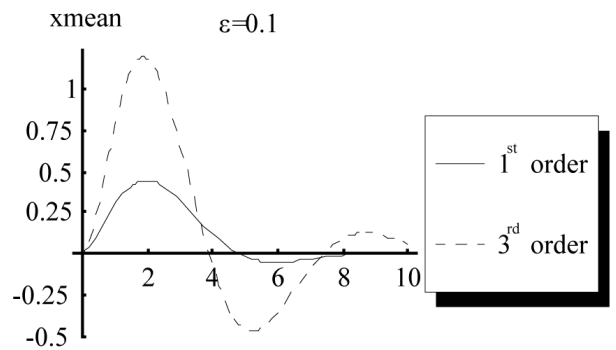

(a)

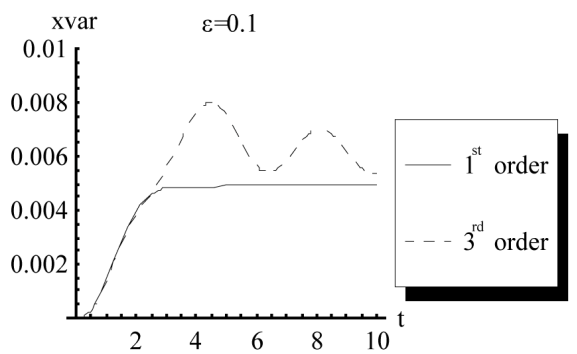

(b)

Figure 6. (a) A comparison between first, second order and the third order of the mean at $\varepsilon=0.1$; (b) Comparison between first, second order and the, third order of the variance at $\varepsilon=\mathbf{0 . 1}$.

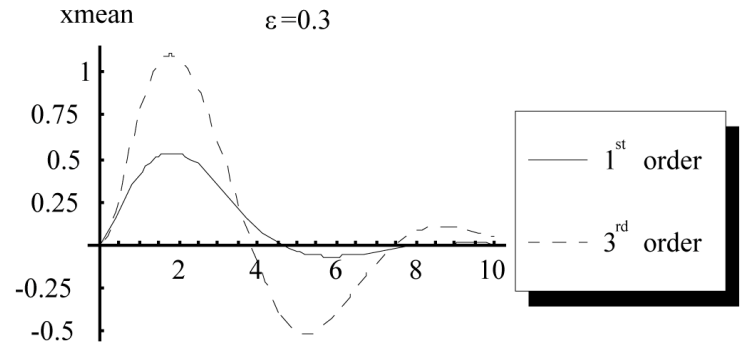

(a)

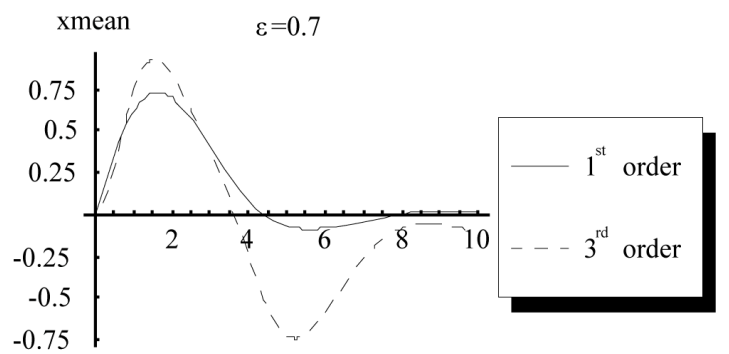

(c)

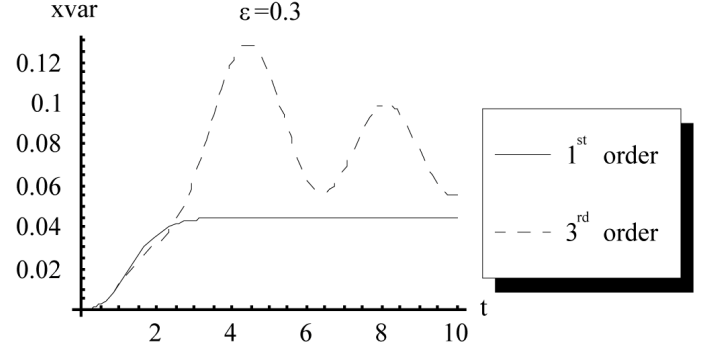

(b)

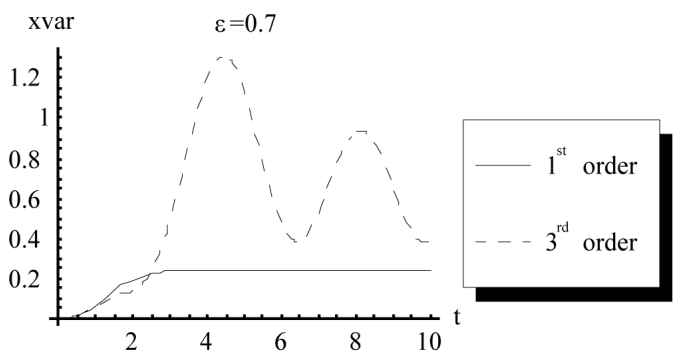

(d)

Figure 7. (a) A comparison between first, second order and the third order of the mean at $\varepsilon=0.3$; (b) A comparison between first, second order and the, third order of the variance at $\varepsilon=0.3$; (c) A comparison between first, second order and the o third order of the mean at $\varepsilon=0.7$; (d) A comparison between first, second order and the third order of the variance at $\varepsilon=0.7$.

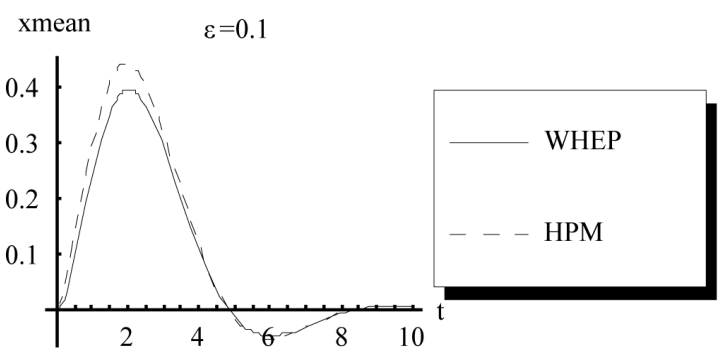

(a)

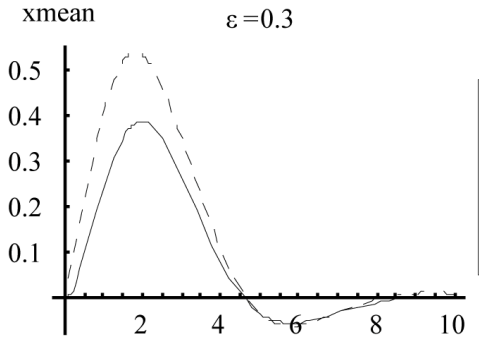

(c)

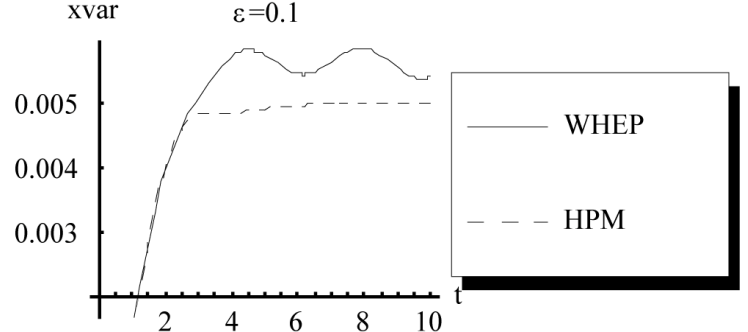

(b)

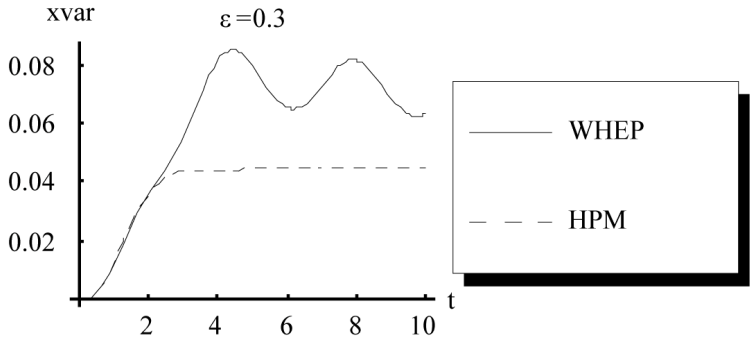

(d) 


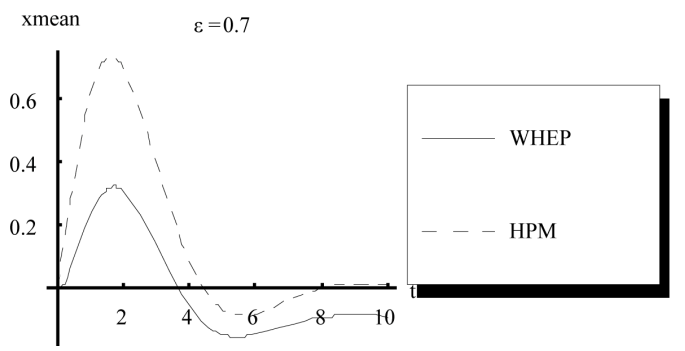

(e)

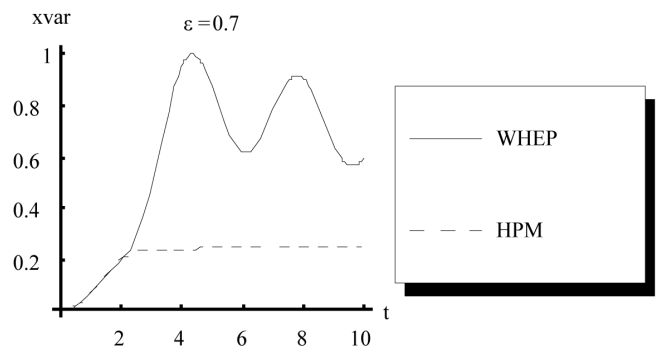

(f)

Figure 8. (a) A comparison between homotopy perturbation and Wiener-Hermite of the mean at $\varepsilon=0.1$; (b) A comparison between homotopy perturbation and Wiener-Hermite of the variance at $\varepsilon=0.1$; (c) A comparison between homotopy perturbation and Wiener-Hermite of the mean at $\varepsilon=0.3$; (d) A comparison between homotopy perturbation and Wiener-Hermite of the variance at $\varepsilon=0.3$; (e) A comparison between homotopy perturbation and Wiener-Hermite of the mean at $\varepsilon=0.7$; (f) A comparison between homotopy perturbation and Wiener-Hermite of the variance at $\varepsilon=0.7$.

This is due to the convergence condition of the WHEP technique which depends on $\varepsilon$. For small values of $\varepsilon$, the WHEP technique converges but after a certain value of $\varepsilon$ it will diverge. The HPM is more accurate for higher values of $\varepsilon$. The HPM has advantages when used in solving differential equations with large nonlinearities.

\section{Conclusion}

The quadratic nonlinear oscillator with stochastic excitation is considered. The solution was obtained using the WHEP technique with different orders and different number of corrections. The HPM is used also with different approximations. The WHEP technique is more efficient but it converges only for certain limit of the nonlinearity strength. The HPM is more difficult in the stochastic differential equations but it is more preferable for higher values of the nonlinearity strength. The two methods are shown to be efficient in estimating the stochastic response of the quadratic nonlinear oscillators.

\section{REFERENCES}

[1] A. Nayfeh, “Problems in Perturbation,” John Wiley, New York, 1993.

[2] S. Crow and G. Canavan, "Relationship between a Wiener-Hermite Expansion and an Energy Cascade,” Journal of Fluid Mechanics, Vol. 41, No. 2, 1970, pp. 387-403. doi:10.1017/S0022112070000654

[3] P. Saffman, "Application of Wiener-Hermite Expansion to the Diffusion of a Passive Scalar in a Homogeneous Turbulent Flow,” Physics of Fluids, Vol. 12, No. 9, 1969, pp. 1786-1798. doi:10.1063/1.1692743

[4] W. Kahan and A. Siegel, "Cameron-Martin-Wiener Method in Turbulence and in Burger's Model: General Formulae and Application to Late Decay," Journal of Fluid Mechanics, Vol. 41, No. 3, 1970, pp. 593-618.

[5] J. Wang and S. Shu, "Wiener-Hermite Expansion and the Inertial Subrange of a Homogeneous Isotropic Turbulence,” Physics of Fluids, Vol. 17, No. 6, 1974, pp. 11301134.
[6] H. Hogge and W. Meecham, "Wiener-Hermite Expansion Applied to Decaying Isotropic Turbulence Using a Renormalized Time-Dependent Base," Journal of Fluid of Mechanics, Vol. 85, No. 2, 1978, pp. 325-347. doi:10.1017/S002211207800066X

[7] M. Doi and T. Imamura, “An Exact Gaussian Solution for Two-Dimensional Incompressible Inviscid Turbulent Flow,” Journal of the Physical Society of Japan, Vol. 46, No. 4, 1979, pp. 1358-1359. doi:10.1143/JPSJ.46.1358

[8] R. Kambe, M. Doi and T. Imamura, "Turbulent Flows Near Flat Plates," Journal of the Physical Society of Japan, Vol. 49, No. 2, 1980, pp. 763-778. doi:10.1143/JPSJ.49.763

[9] A. J. Chorin, “Gaussian Fields and Random Flow,” Journal of Fluid of Mechanics, Vol. 63, No. 1, 1974, pp. 2132. doi:10.1017/S0022112074000991

[10] Y. Kayanuma, "Stochastic Theory for Non-Adiabatic Crossing with Fluctuating Off-Diagonal Coupling," Journal of the Physical Society of Japan, Vol. 54, No. 5, 1985, pp. 2037-2046. doi:10.1143/JPSJ.54.2037

[11] M. Joelson and A. Ramamonjiarisoa, "Random Fields of Water Surface Waves Using Wiener-Hermite Functional Series Expansions," Journal of Fluid of Mechanics, Vol. 496, 2003, pp. 313-334. doi:10.1017/S002211200300644X

[12] C. Eftimiu, "First-Order Wiener-Hermite Expansion in the Electromagnetic Scattering by Conducting Rough Surfaces," Radio Science, Vol. 23, No. 5, 1988, pp. 769-779. doi:10.1029/RS023i005p00769

[13] N. J. Gaol, "Scattering of a TM Plane Wave from Periodic Random Surfaces,” Waves Random Media, Vol. 9, No. 11, 1999, pp. 53-67.

[14] Y. Tamura and J. Nakayama, "Enhanced Scattering from a Thin Film with One-Dimensional Disorder," Waves in Random and Complex Media, Vol. 15, No. 2, 2005, pp. 269-295.

[15] Y. Tamura and J. Nakayama, "TE Plane Wave Reflection and Transmission from One-Dimensional Random Slab,” IEICE Transactions on Electronics, Vol. E88-C, No. 4, 2005, pp. 713-720.

[16] N. Skaropoulos and D. Chrissoulidis, "Rigorous Application of the Stochastic Functional Method to Plane Wave 
Scattering from a Random Cylindrical Surface,” Journal of Mathematical Physics, Vol. 40, No. 1, 1999, pp. 156168. doi:10.1063/1.532766

[17] A. Jahedi and G. Ahmadi, “Application of Wiener-Hermite Expansion to Non-Stationary Random Vibration of a Duffing Oscillator," Journal of Applied Mechanics, Transactions ASME, Vol. 50, No. 2, 1983, pp. 436-442.

[18] I. I. Orabi and G. Ahmadi, "Functional Series Expansion Method for Response Analysis of Nonlinear Systems Subjected to Ransom Excitations,” International Journal of Nonlinear Mechanics, Vol. 22, No. 6, 1987, pp. 451465. doi:10.1016/0020-7462(87)90036-9

[19] I. I. Orabi, "Response of the Duffing Oscillator to a NonGaussian Random Excitation," Journal of Applied Mechanics, Transaction of ASME, Vol. 55, No. 3, 1988, pp. 740-743.

[20] E. Abdel Gawad, M. El-Tawil and M. A. Nassar, "Nonlinear Oscillatory Systems with Random Excitation," Modeling, Simulation and Control B, Vol. 23, No. 1, 1989, pp. 55-63.

[21] I. I. Orabi and G. Ahmadi, "New Approach for Response Analysis of Nonlinear Systems under Random Excitation," American Society of Mechanical Engineers, Design Engineering Division (Publication) DE, Vol. 37, 1991, pp. 147-151.

[22] E. Gawad and M. El-Tawil, "General Stochastic Oscillatory Systems," Applied Mathematical Modelling, Vol. 17, No. 6, 1993, pp. 329-335. doi:10.1016/0307-904X(93)90058-O

[23] M. El-Tawil and G. Mahmoud, "The Solvability of Parametrically Forced Oscillators Using WHEP Technique," Mechanics and Mechanical Engineering, Vol. 3, No. 2, 1999, pp. 181-188.

[24] Y. Tamura and J. Nakayama, “A Formula on the Hermite Expansion and Its Aoolication to a Random Boundary Value Problem," IEICE Transactions on Electronics, Vol. E86-C, No. 8, 2003, pp. 1743-1748.

[25] M. El-Tawil, "The Application of WHEP Technique on Stochastic Partial Differential Equations,” International Journal of Differential Equations and Applications, Vol. 7, No. 3, 2003, pp. 325-337.

[26] Y. Kayanuma and K. Noba, "Wiener-Hermite Expansion Formalism for the Stochastic Model of a Driven Quantum System," Chemical Physics, Vol. 268, No. 1-3, 2001, pp. 177-188. doi:10.1016/S0301-0104(01)00305-6

[27] O. Kenny and D. Nelson, "Time-Frequency Methods for Enhancing Speech,” Proceedings of SPIE-The International Society for Optical Engineering, Vol. 3162, 1997, pp. 48-57.

[28] E. Isobe and S. Sato, "Wiener-Hermite Expansion of a Process Generated by an Ito Stochastic Differential Equations," Journal of Applied Probability, Vol. 20, No. 4, 1983, pp. 754-765. doi:10.2307/3213587

[29] R. Rubinstein and M. Choudhari, "Uncertainty Quantifi- cation for Systems with Random Initial Conditions Using Wiener-Hermite Expansions,” Studies in Applied Mathematics, Vol. 114, No. 2, 2005, pp. 167-188. doi:10.1111/j.0022-2526.2005.01543.x

[30] T. Imamura, W. Meecham and A. Siegel, "Symbolic Calculus of the Wiener Process and Wiener-Hermite Functionals,” Journal of Mathematical Physics, Vol. 6, No. 5, 1983, pp. 695-706. doi:10.1063/1.1704327

[31] J. H. He, “Homotopy Perturbation Technique,” Computer Methods in Applied Mechanics and Engineering, Vol. 178, 1999, pp. 257-292. doi:10.1016/S0045-7825(99)00018-3

[32] J. H. He, "A Coupling Method of a Homotopy Technique and a Perturbation Technique for Nonlinear Problems," International Journal of Nonlinear Mechanics, Vol. 35, 2000, pp. 37-43. doi:10.1016/S0020-7462(98)00085-7

[33] J. H. He, "Homotopy Perturbation Method: A New Nonlinear Analytical Technique," Applied Mathematics and Computation, Vol. 135, 2003, pp. 73-79.

[34] J. H. He, "The Homotopy Perturbation Method for Nonlinear Oscillators with Discontinuities," Applied Mathematics and Computation, Vol. 151, 2004, pp. 287-292.

[35] J. H. He, "Some Asymptotic Methods for Strongly Nonlinear Equations," International Journal of Modern Physics B, Vol. 20, No. 10, 2006, pp. 1141-1199.

[36] S. J. Liao, "On the Proposed Homotopy Analysis Techniques for Nonlinear Problems and Its Applications," Ph.D. Dissertation, Shanghai Jiao Tong University, 1992.

[37] S. J. Liao, "Beyond Perturbation: Introduction to the Homotopy Analysis Method," Chapman \& Hall\CRC Press, Boca Raton, 2003.

[38] S. J. Liao, “An Approximate Solution Technique Which Does Not Depend upon Small Parameters: A Special Example," International Journal of Nonlinear Mechanics, Vol. 30, 1995, pp. 371-380. doi:10.1016/0020-7462(94)00054-E

[39] T. Hayat, M. Khan and S. Asghar, "Homotopy Analysis of MHD Flows of an Oldroyd 8-Constant Fluid," Acta Mechanica, Vol. 168, 2004, pp. 213-232. doi:10.1007/s00707-004-0085-2

[40] S. Asghar, M. Mudassar and M. Ayub, "Rotating Flow of a Third Fluid by Homotopy Analysis Method," Applied Mathematics and Computation, Vol. 165, 2005, pp. 213-221. doi:10.1016/j.amc.2004.04.047

[41] S. P. Zhu, "A Closed Form Analytical Solution for the Valuation of Convertible Bonds with Constant Dividend Yield,” ANZIAM Journal, Vol. 47, No. Part 4, 2006, pp. 477-494.

[42] M. A. El-Tawil and A. S. Al-Johani, "Approximate Solution of a Mixed Nonlinear Stochastic Oscillator," Computers \& Mathematics with Applications, Vol. 58, No. 11-12, 2009, pp. 2236-2259. doi:10.1016/j.camwa.2009.03.057 